Research Article

\title{
A Noise-Immune Kalman Filter and Modelling for Pedestrian Traffic Loads
}

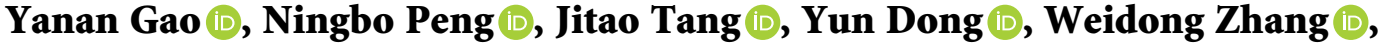 \\ Peng Zhang $\mathbb{D}$, Jiarui Chen $\mathbb{D}$, and Ye Zhu $\mathbb{D}$
}

Faculty of Architecture and Civil Engineering, Huaiyin Institute of Technology, Huaian 223001, China

Correspondence should be addressed to Ningbo Peng; pengnb10@lzu.edu.cn

Received 2 June 2020; Revised 10 September 2020; Accepted 5 November 2020; Published 24 November 2020

Academic Editor: Wai-on Wong

Copyright (c) 2020 Yanan Gao et al. This is an open access article distributed under the Creative Commons Attribution License, which permits unrestricted use, distribution, and reproduction in any medium, provided the original work is properly cited.

\begin{abstract}
The definition of design load with walking crowd excitation on these slender structures is a significant problem to human-induced vibration. To capture the characteristics of walking crowd loads, this article researches both the ground reaction force and ground reaction moment for 36 healthy adults. Firstly, a oscillate system modeling walking leg is used to build a governing equation, which further transformed into the discrete state space. Then the Kalman method is applied to filter the noises for the measured ground reaction force, which can well remove the noises hiding in the measured signals. In addition, the Fourier series are used to model the ground reaction force and ground reaction moment, and the first six corresponding coefficients are obtained and analyzed. This work comprehensively explores the excitation force and moment from walking pedestrian feet. The result of this study provides the reference of load design for these slender structures such as footbridges, grandstands, or stations under crowd excitation.
\end{abstract}

\section{Introduction}

The vibrations of slender structures induced by excitation due to pedestrian traffic remain a large challenge. Uncertainties associated with human locomotive behavior and the dynamic properties of the human body result in considerable complexity in describing the vibrations resulting from pedestrians. In order to explore the effect of pedestrian excitation on structures, Rainer and Pernica [1] first measured the dynamic load factors (DLFs) during walking, running, and jumping using an instrumented platform. The time history of force of walking people [2] showed that the ground reaction force (GRF) from walking excitation produced an M-shaped curve, which was fitted using a Fourier function [3]. Based on this research, Fan et al. [4] constructed a standard GRF model using the least-squares method. Although the GRF model was able to describe the excitation from walking pedestrians, the contributions from the pedestrian's dynamic properties were not included. Kerr and Bishop [5] investigated the differences between humaninduced loadings on a floor with that generated whilst ascending or descending a staircase. Hsiang and Chang [6] measured vertical ground reaction forces during fifteen gait speed and load carrying (five load positions and three speeds), which concluded that both speed and loading conditions can prompt the gait control system to adjust the gait pattern to maintain successful gait. Kala et al. [7] measured the vertical ground reaction forces and obtained the first four dynamic coefficients as $0.32,0.09,0.12$, and 0.01 under $1.55 \mathrm{~Hz}$ step frequency and $1.4 \mathrm{~m} / \mathrm{s}$ speed. Besides, some measurements on lateral ground reaction forces have also been implemented. For example, Ricciardelli and Pizzimenti [8] investigated the lateral forces exerted by walkers on a fixed floor and calibrated deterministic and stochastic lateral loading models on a footbridge; Ingolfsson et al. [9] presented the experimental analysis on lateral forces generated by the single pedestrians during continuous walking on a treadmill; Bocian et al. [10] carried a novel experimental setup for obtaining lateral forces by avoiding the implications of artificiality and allowing for unconstrained gaits. In addition, an elaborated self-sustained oscillator [11] was used to predict the lateral walking forces induced by walking 
pedestrians. Based on these measurement results, the walking forces have been extensively applied on the vibration analysis of these structures bearing crowd loads. Mouring and Ellingwood [12] early analyzed the influence of crowd loads on the dynamic response of floor systems. Pedersen and Frier [13] presented a stochastic modeling method, which was adopted for quantifying the probability of exceeding various vibrations in a footbridge design. $\mathrm{Li}$ et al. [3] proposed a vibration control method by using multiple tuned mass dampers carrying on the crowd-induced random vibrations of the footbridge. Besides, walking pedestrian can cause vibrations, and torsional shakes have also been observed [14]. However, the past traditional measurements on walking loads have mostly focused on the vertical ground reaction forces. The reaction forces from walking foot contain more information such as moments, but it is a lack of exploration.

This work comprehensively investigates the three-dimensional spatial ground reaction force (GRF) and ground reaction moment (GRM) induced by walking pedestrian feet and extracts the corresponding mechanical characteristics, based on the measurements with a total of 36 healthy young adults. In Section 2, an oscillator modeling with the walking single leg and the corresponding dynamic governing equation is developed to describe the measured forces. A systematical state equation is further developed so that its state equation and measured equations consider the contributions of the inertia, damping, stiffness, and foot forces. Based on the equation, the Kalman filtering method concentrating on the walking leg model is applied to remove the adverse noises from the measured rough data so as to improve the signal-to-noise ratio on the measured ground reaction forces. Some different noise level data are used to verify the effectiveness of the filter. In Section 3, a force platform is used to generate the GRF and GRM excitation from the 36 young adults, which comprised of 11 females and 25 males. The body and walking characteristics of the testers including mass, height, and step rate are statistically analyzed to present the mean, variances, and correlations among them. Based on the measurements, the Fourier series are applied to describe the measured forces and moments, and the corresponding coefficients including dynamic load factor (DLF) and dynamic moment factor (DMF) are presented for analysis. Furthermore, the statistical characteristics of DLF and DMF are comprehensively studied, so the loading models from the ground reaction forces and moments are established to describe the standard forcing models on these structures enduring on the walking crowd excitation.

\section{Kalman Filtering Process}

2.1. System State Equations. In order to establish the Kalman filter [15] for ground reaction force (GRF), the pedestrian is simplified as a mechanical model with one upper body and lower moving leg (Figure 1). The upper body occupies a lump mass $m$, and the lower leg models as a mass-less spring-damping oscillator. The stiffness and damping parameters of the leg are $k$ and $c$, respectively. The GRF $F$ is

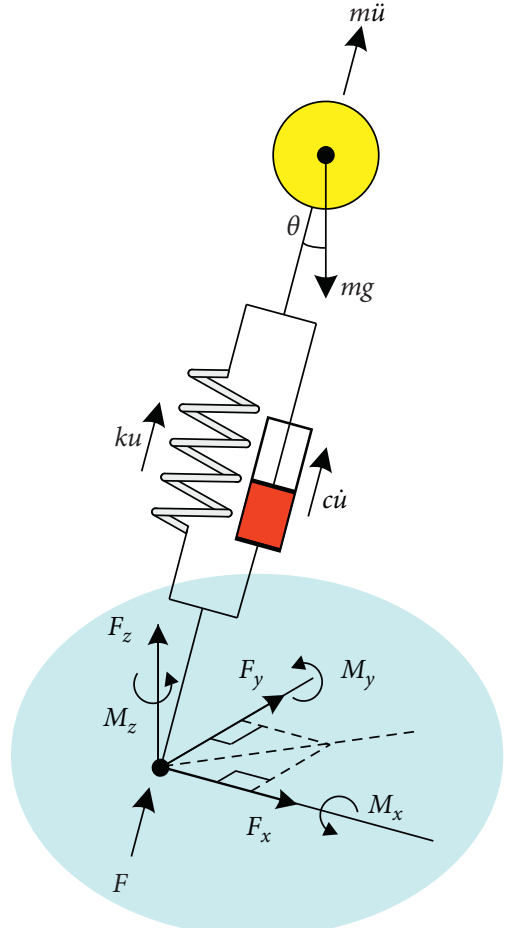

FIGURE 1: Forcing diagram of single-leg model.

parallel to the axis of the leg, and it can be decomposed into three components as longitudinal GRF $F_{x}$, lateral GRF $F_{y}$, and vertical GRF $F_{z}$, to be measured. Besides, the ground reaction moments (GRMs) from foot in $x, y$, and $z$ directions are longitudinal GRM $M_{x}$, lateral GRM $M_{y}$, and vertical GRM $M_{z}$, respectively. The axial compression displacement of the leg is denoted by $u$. The intersection angle between the leg axial and gravity directions is the sign $\theta$, and the governing equation of the simplified mechanical system can be established as follows:

$$
m \ddot{u}(t)+c \dot{u}(t)+k u(t)=f(t),
$$

where the variables $\ddot{u}$ and $\dot{u}$ are the corresponding leg axial acceleration and velocity, respectively, and $f(t)$ means the external force along with the axis of the leg induced by body weight and it is shown in the following equation:

$$
f(t)=m g \cos \theta(t),
$$

where $g$ means the gravitational acceleration and its value is $9.81 \mathrm{~m} / \mathrm{s}^{2}$ and the $\cos \theta$ means the projection ratio of the vertical GRF $F_{z}$ to the synthesis force $F$, which is calculated as follows:

$$
\cos \theta(t)=\frac{F_{z}(t)}{F(t)}=\frac{F_{z}(t)}{\sqrt{F_{x}(t)^{2}+F_{y}(t)^{2}+F_{z}(t)^{2}}} .
$$

The governing equation of motion of the leg system in equation (1) can be transformed into a state-space equation as follows:

$$
\dot{X}(t)=\mathbf{A} X(t)+\mathbf{B} f(t),
$$


where $X(t), \mathbf{A}$, and $\mathbf{B}$, respectively, are the state vector, state matrix, and input-to-state matrix under a continuous state condition, which is shown as follows:

$$
\begin{aligned}
X(t) & =\left\{\begin{array}{c}
u(t) \\
\dot{u}(t)
\end{array}\right\}, \\
A & =\left[\begin{array}{cc}
0 & 1 \\
-m^{-1} k & -m^{-1} c
\end{array}\right], \\
B & =\left[\begin{array}{c}
0 \\
m^{-1}
\end{array}\right] .
\end{aligned}
$$

Multiplying the matrix $e^{-\mathrm{A} t}$ on both sides of equation (4) would result in

$$
e^{-\mathbf{A} t}[\dot{X}(t) t-n A q X h(t)]=e^{-\mathbf{A} t} \mathbf{B} f(t) .
$$

Substitute the identical equation $(\mathrm{d} / \mathrm{d} t)\left[e^{-\mathbf{A} t} X(t)\right]=e^{-\mathbf{A} t}[\dot{X}(t) t-n A q X h(t)]$ into equation (6). Then, integrating equation (6) between the ranges from the time step $t_{0}$ to $t_{i}\left(t_{0}<t_{i}\right)$ would able to obtain the following equation:

$$
X(t)=e^{\mathbf{A}\left(t-t_{0}\right)} X\left(t_{0}\right)+\int_{t_{0}}^{t} e^{\mathbf{A}(t-\tau)} \mathbf{B} f(\tau) \mathrm{d} \tau .
$$

Define $\Phi\left(t, t_{0}\right)=e^{\mathbf{A}\left(t-t_{0}\right)}$ and it is substituted into equation (7) and consider the $t_{i+1}$ state can be changed as follows:

$$
X\left(t_{i+1}\right)=\boldsymbol{\Phi}\left(t_{i+1}, t_{i}\right) X\left(t_{i}\right)+\int_{t_{i}}^{t_{i+1}} \boldsymbol{\Phi}\left(t_{i+1}, \tau\right) \mathbf{B} f(\tau) \mathrm{d} \tau .
$$

The sampling frequency is assumed to be a constant, the input is also assumed to be linearly changed in the time domain of $\left[t_{i}, t_{i+1}\right]$, and it is approximately expressed as follows:

$$
f(\tau)=f_{i}+\frac{f_{i+1}-f_{i}}{\Delta t}\left(\tau-t_{i}\right),
$$

where $\Delta t=t_{i+1}-t_{i}$ is the sampling interval of a measured signal.

Substituting equation (9) into equation (8) will result in

$$
X_{i+1}=e^{\mathbf{A} \Delta t} X_{i}+\int_{t_{i}}^{t_{i+1}} e^{\mathbf{A}\left(t_{i+1}-\tau\right)} \mathbf{B}\left[f_{i}+\frac{f_{i+1}-f_{i}}{\Delta t}\left(\tau-t_{i}\right)\right] \mathrm{d} \tau \text {. }
$$

Define $\left(q=\tau-t_{i}\right)$ and substituting it into equation (10) would obtain the following equation:

$$
\begin{aligned}
X_{i+1}= & e^{\mathbf{A} \Delta t} X_{i}+\int_{0}^{\Delta t} e^{\mathbf{A}(\Delta t-q)} \mathbf{B}\left[f_{i}+\frac{f_{i+1}-f_{i}}{\Delta t} q\right] \mathrm{d} q, \\
= & e^{\mathbf{A} \Delta t} X_{i}+\left(e^{\mathbf{A} \Delta t}-\mathbf{I}\right) \mathbf{A}^{-1} \mathbf{B} f_{i} \\
& -\left[\Delta t+\left(\mathbf{I}-e^{\mathbf{A} \Delta t}\right) \mathbf{A}^{-1}\right] \mathbf{A}^{-1} \mathbf{B} \frac{f_{i+1}-f_{i}}{\Delta t} .
\end{aligned}
$$
that

The time interval $\Delta t$ is assumed to be enough small so hat

$$
e^{\mathbf{A} \Delta t} \approx \mathbf{I}+\mathbf{A} \Delta t .
$$

After substituting equation (12) into equation (11), equation (11) can be rewritten as follows:

$$
X_{i+1}=\Psi X_{i}+\Gamma f_{i},
$$

where $\left(\Psi=e^{\mathbf{A} \Delta t}\right)$ and $(\Gamma=\mathbf{B} \Delta t)$, respectively, are the state matrix and state vector under a discrete state condition.

The equilibrium equation in the lower supported point can be described as follows:

$$
F=\mathbf{H} X
$$

where $\mathrm{H}=[k c]$ is a measure matrix.

Combining equations (13) and (14) and the noises, the system state equation of Kalman filter can be obtained as follows:

$$
\left\{\begin{array}{l}
X_{i+1}=\boldsymbol{\Psi} X_{i}+\Gamma f_{i}+w_{i} \\
F_{i+1}=\mathbf{H} X_{i+1}+v_{i+1}
\end{array}\right.
$$

where $w_{i}$ and $v_{i+1}$ are process noise and measurement noise, which satisfy normal distribution as follows:

$$
\begin{aligned}
w_{i} & \sim N(0, \mathbf{Q}), \\
v_{i} & \sim N(0, \mathbf{R}),
\end{aligned}
$$

where $\mathbf{Q}=E\left(w_{i} w_{i}^{T}\right)$ and $\mathbf{R}=E\left(v_{i} v_{i}^{T}\right)$ are the covariances of process noise and measurement noise, respectively.

In the system state equations of equation (14), it is noted that $X_{i} \in \mathbb{R}^{2}$ and $F_{i+1} \in \mathbb{R}^{1}$ represent the 2-dimensional state vector and 1-dimensional measurement vector, respectively. $\psi$ is the transition matrix and $\mathbf{H}$ is the measurement matrix. Compared with other filter methods, the Kalman filtering can effectively deduce the corruption based on the minimum mean square error criterion. In the algorithm, it needs to be time-discrete so as to realize the filtering process in a computer, and its solving flowchart is indicated in Figure 2 from the Appendix. The details with state estimation of $X$, estimation error $\mathcal{E}$, estimation covariance $P$, and gain matrix $\mathbf{K}$ in each time point are formulaically presented in the Appendix. It is noted that the parameters including iteration number $i=0$, initial displacement $u$, body mass $m$, leg damping $c$, leg stiffness $k$, process noise covariance matrix $\mathbf{Q}$, measurement noise covariance matrix $\mathbf{R}$, and time computing interval $\Delta t$ are prior defined and input. The Kalman filter is a linear system due to its development on the linear governing equation in equation (1). So the filter can only deal with linear filtering problems, and it is not suitable for some more complex nonlinear problems. In the iteration solving process, the initial covariance $P_{0 \mid 0}$ and state estimation $\widehat{X}_{0 \mid 0}$ of $X$ also need to be defined in advance and corresponding equation details on the filtering process are shown in the Appendix. The filtering result can be extracted in equation (A.9) and Figure 2 gives the flow diagram of the Kalman filtering process on measured GRFs.

2.2. Verification on Kalman Filter. Before treating measured GRFs, some artificial ground reaction forces generated by a bipedal robot model are applied to filter under different 


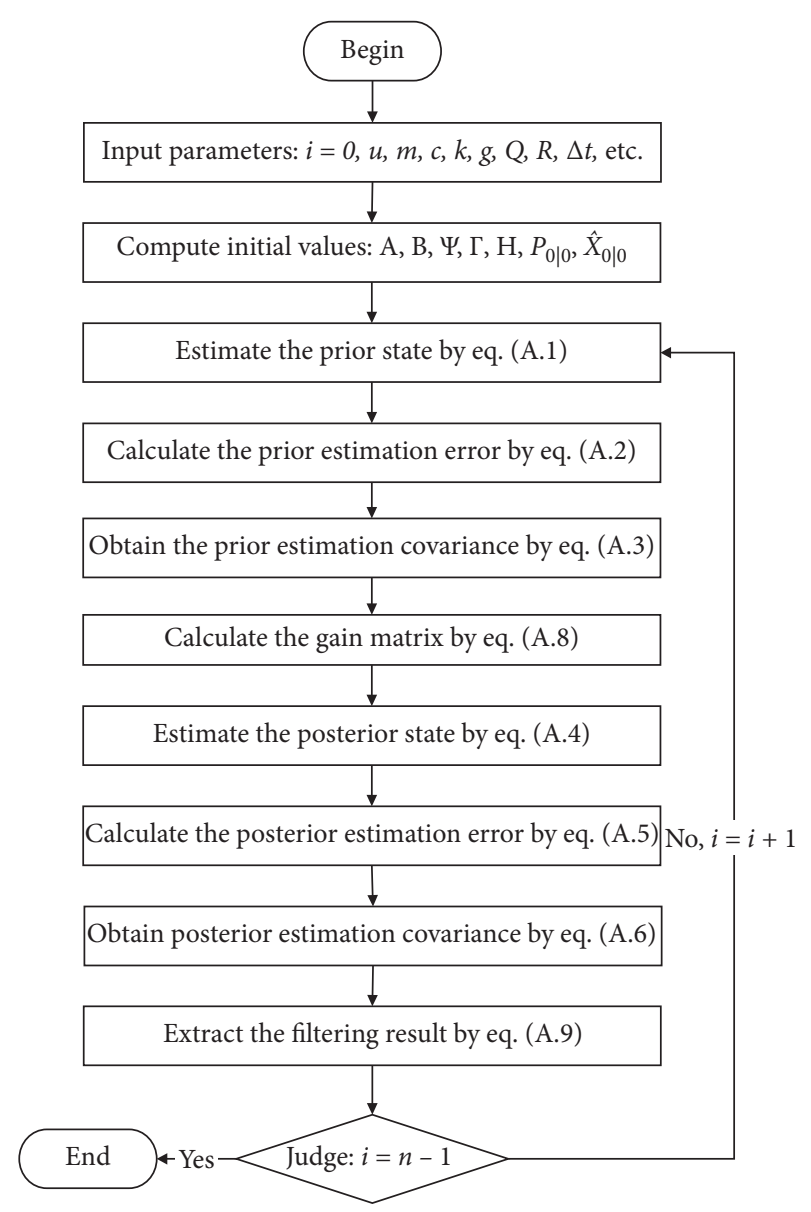

FIgURE 2: Flow diagram of Kalman filtering on measured GRFs.

noise levels. The bipedal robot occupies $80 \mathrm{~kg}$ mass and other corresponding parameters can be found in the study of Gao and his cooperator [16]. The GRFs including $F_{x}, F_{y}$, and $F_{z}$ are obtained from the typical simulation of the $3 \mathrm{D}$ walking process [16]. Figure 3 gives the filtering results and the vertical ordinate force $F$ is divided by the body weight $G$. The blue and red lines are the untreated and filtered lines, respectively. There present four noise levels with $0 \%, 5 \%, 10 \%$, and $20 \%$ of the synthetic force $F$ with $F_{x}, F_{y}$, and $F_{z}$, whose corresponding filtering results are indicated in Figures 3(a) to $3(\mathrm{~d})$, respectively. It is noted that the processed lines always situate the centers of the rough original data, and the filtered lines are very close to the shape of the noiseless composite $F$ in Figure 3(a). The error between the filtered data and noiseless signal in Figure 4(a) shows that the increase of noise level would not cause a significant increase in the errors. The relative error ranges with different noise levels are mainly included in the range between $-20 \%$ and $20 \%$. The absolute errors are also given in Figure 4(b) and the lower and upper boundary of the rectangular regions denote the $25 \%$ and $75 \%$ of the total contents. The Kalman filter can perfectly find the accurate GRF without noise. Along with the increases of noise levels, the absolute errors also increased. However, the maximum error is less than $0.3 \mathrm{~N}$ and the proposed Kalman filter has good denoising properties on treating GRF data. In addition, the filter method always makes the filtered data approach to the accurate signal, which indicates the Kalman filter has well convergence and robustness.

\section{Treatment on Measurements}

A force platform in Figure 5 is used to generate the GRF and GRM excitation from the 36 young adults, which comprised of 11 females and 25 males. The measurement platform includes one force platform and two footpaths [17]. The $0.6 \mathrm{~m}$ square force platform has a thickness of $0.1 \mathrm{~m}$. The entrance footpath is $1.5 \mathrm{~m}$ long and $0.6 \mathrm{~m}$ wide with a $0.5 \mathrm{~m}$ long slope for gait adjustment as the pedestrian enters the footpath. The length and width of the exit footpath are $1.0 \mathrm{~m}$ and $0.6 \mathrm{~m}$, respectively. The thicknesses of both footpaths are the same as that of the force platform. All participants maintain natural relaxation as far as possible in the test process. When a subject leaves the exit footpath, he/she turns to walk towards the entrance footpath until the test ends. In the walking tests, all testers remained as natural and relaxed as possible.

The ages of these participants are inside the range of 18 and 21 years. Their physical indexes include body mass, height, and step rates as shown in Figure 6. The body mass and height approximately obey normal distribution, and their corresponding means are $67.7 \mathrm{~kg}$ and $172.9 \mathrm{~cm}$, and standard deviations are $11.0 \mathrm{~kg}$ and $9.2 \mathrm{~cm}$, respectively. The nonlinear fit based on the least-square method between the body mass and height is indicated in Figure 7, whose relationship is $h=102.7+1.6 \mathrm{~m}-0.008 \mathrm{~m}^{2}$, which indicates that the height is slightly increased along with the increase of body mass. However, the increased range is decreased along with the increase of body mass. The variables $m$ and $h$ are body mass and height, respectively. The larger body mass tends to have taller height. Although a metronome is applied to guide the step rate of the walking participants, they have some difficulties to follow beat rates. All testers walk as slow as possible to keep a relaxed mental state, which presents in the distribution range of the step rates occupying the wider range of 0.6 to $2.4 \mathrm{~Hz}$. Walking step rates $\left(f_{\mathrm{s}}\right)$ approximately reveal the normal statistical distribution with the $1.3 \mathrm{~Hz}$ mean and $0.35 \mathrm{~Hz}$ standard deviation as shown in Figure 8 .

The collecting device is a measure-analysis system on multidimensional forces produced by the Anhui Aili Intelligent Technology Limited-liability Company. The sampling frequency is $1000 \mathrm{~Hz}$. The leg stiffness $k$ and damping ratio are input as $20 \mathrm{kN} / \mathrm{m}$ and $8 \%$, respectively. The observation on the untreated signals indicates that the initial measured data mixed a lot of noises mainly due to the interference of electromagnetic equipment and structural vibration. All measured GRFs including $F_{x}, F_{y}$, and $F_{z}$ are filtered by the proposed Kalman filter method. Figure 9 gives the typical filtering results from a male tester with $69 \mathrm{~kg}$ mass and $182 \mathrm{~cm}$ height. It is noted that the peak values from normalized GRF $F_{x} / G$ in longitudinal, $F_{y} / G$ in lateral, and $F_{z} / G$ in vertical directions are close to $0.2,0.1$, and 1.1, respectively. The shape of $F_{x}$ is firstly concave and then convex. The shapes of $F_{y}$ and $F_{z}$ are similar and look likely as the back of camel. The normalized GRMs in $x, y$, and $z$ are shown in Figures 9(d) to 9(f), respectively. It is noted that the peaks from 


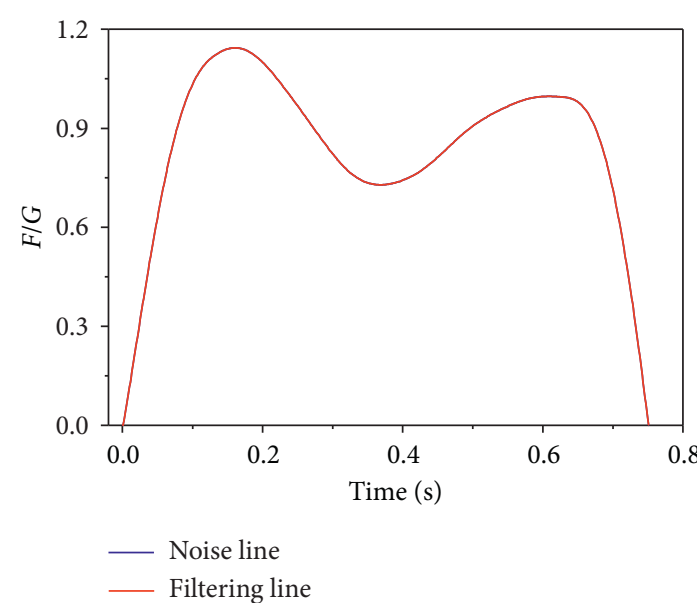

(a)

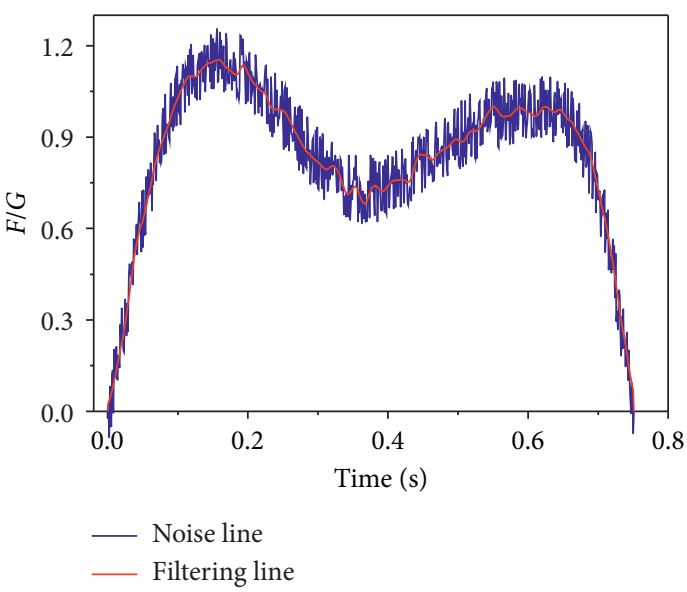

(c)

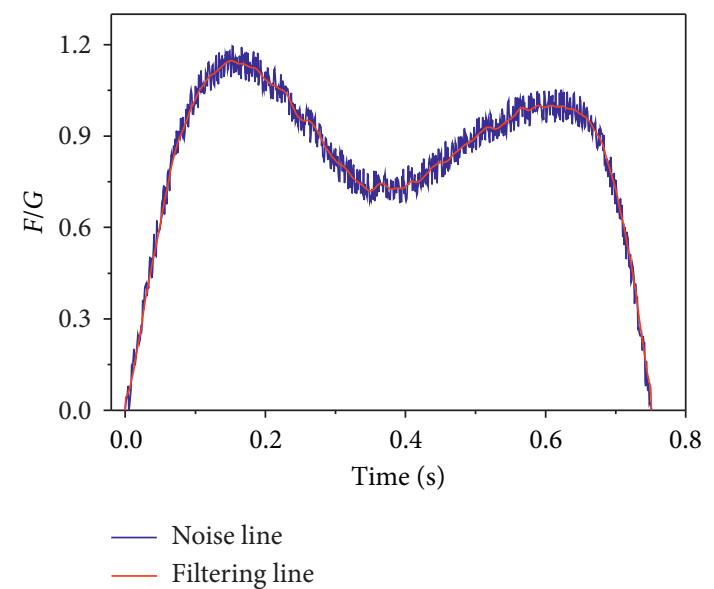

(b)

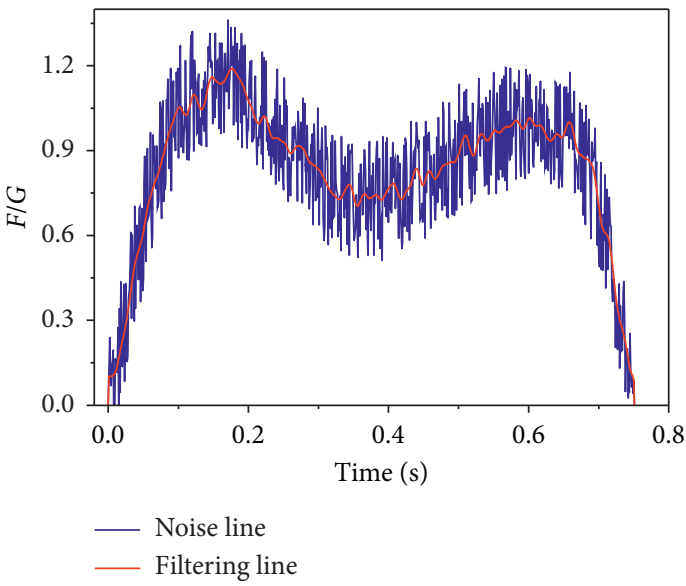

(d)

FiguRE 3: Filtering results with different noise levels: (a) without noise; (b) 5\% noise; (c) 10\% noise; (d) $20 \%$ noise.

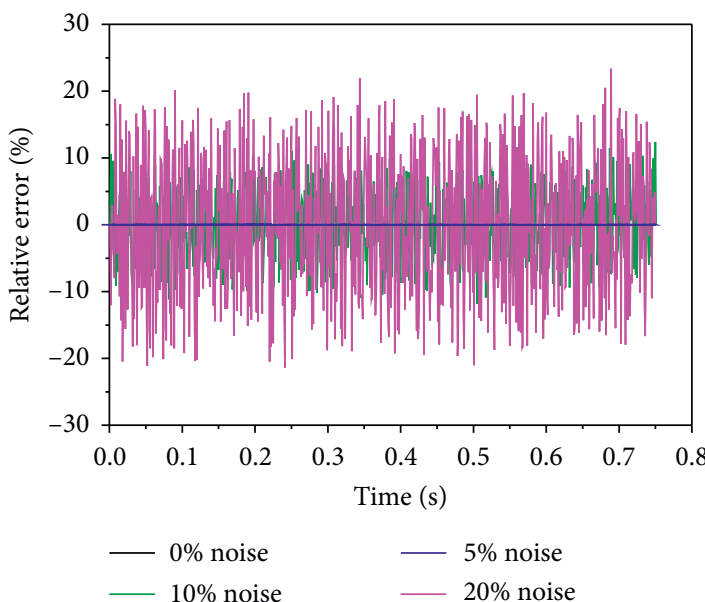

(a)

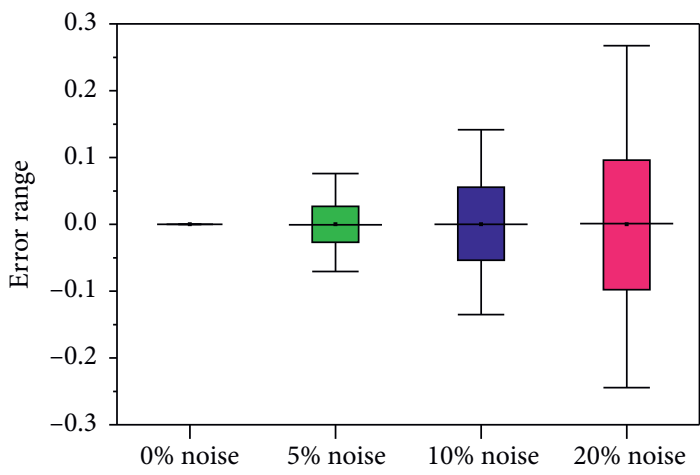

(b)

FiguRE 4: Error in the filtering process with different noise levels: (a) relative error and (b) absolute error.

normalized moments $M_{x} / G$ in longitudinal, $M_{y} / G$ in lateral, and $M_{z} / G$ in vertical directions are close to $0.12,0.3$, and 0.04 , respectively. The peaks from longitudinal and lateral moments are far larger than the vertical moment, which means that walking foot mainly generates longitudinal and lateral moments. 


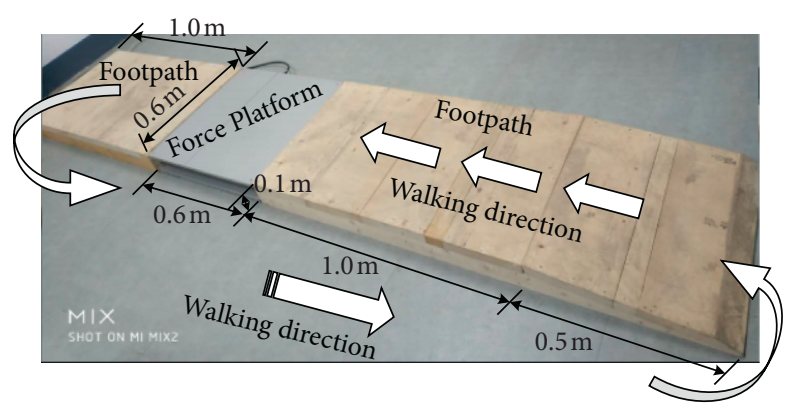

FIGURE 5: Measurement platform on ground reaction forces.

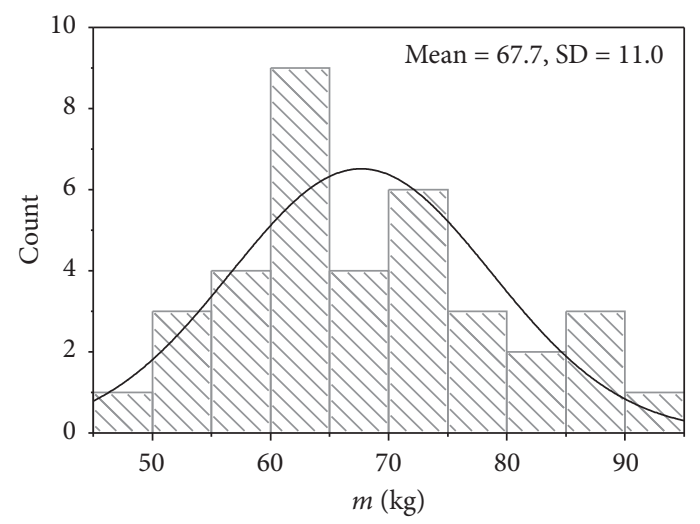

(a)

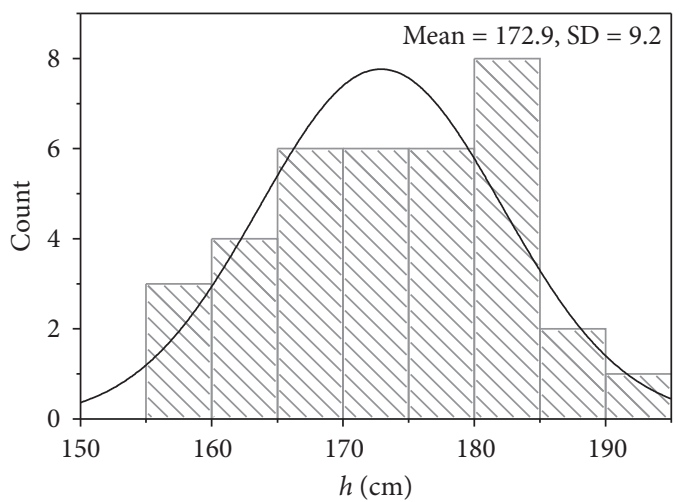

(b)

FIGURE 6: Statistical characteristics of body mass and height.

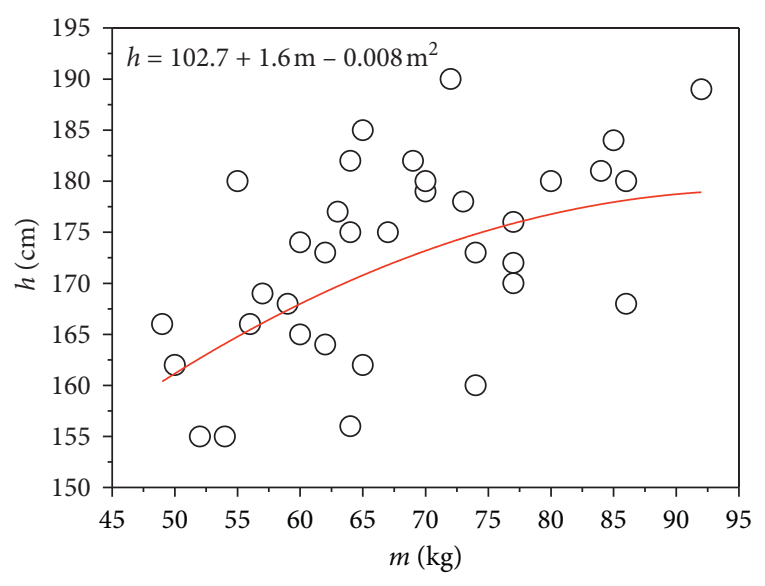

FIGURE 7: Fitting relationship between body mass and height.

In order to find the influential effects of the step rate of walking, the statistical correlation between the step rate and the peaks of normalized measured forces by body gravity $G$ was comprehensively analyzed. Table 1 lists the coefficients of all the fitting relationships. The maximum intercept value of the peak Fz/G (Figure 10(c)) is 1.04, which indicates that structure under a walking pedestrian endures the worst force impact in the vertical direction. However, the intercepts with moments from $\mathrm{x}$ and $\mathrm{y}$ directions corresponding

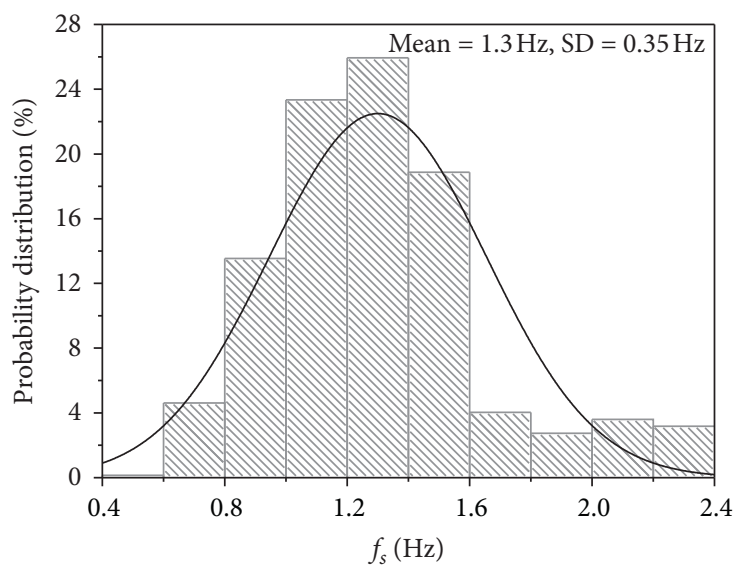

FIgURE 8: Probability distribution of step rate.

to 0.11 in Figure 11(a) and to 0.14 in Figure 11(b) are much larger than those from the $\mathrm{z}$ direction corresponding to 0.03 in Figure 11(c). Pedestrian are more likely to trigger the torsions in the longitudinal and lateral directions than in the vertical direction for a structure. The maximum slope with GRFs is 0.027 in the vertical direction, which implies that the increase of step rate has the most significant impact on the vertical excitation. The larger speed would cause the larger excitation force, while the slope impacts from GRMs are different from the GRFs. The slope values with GRFs 


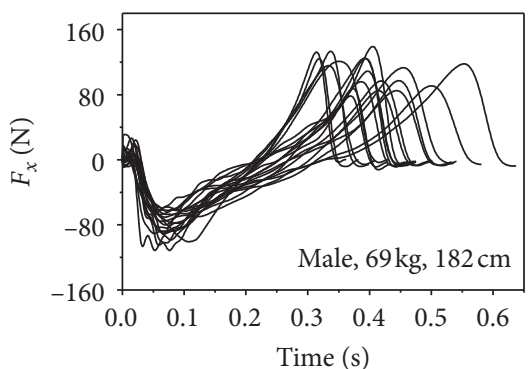

(a)

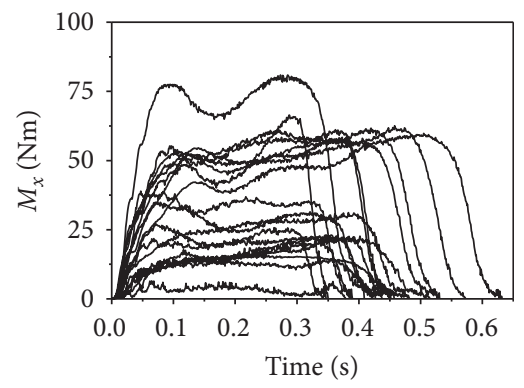

(d)

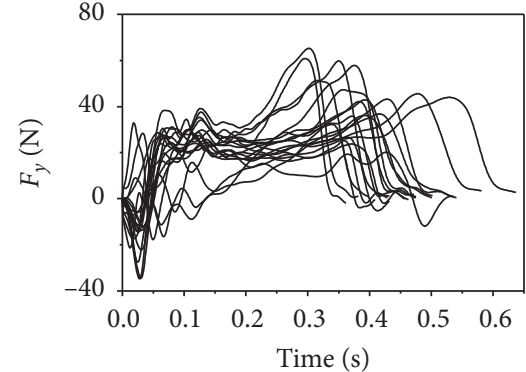

(b)

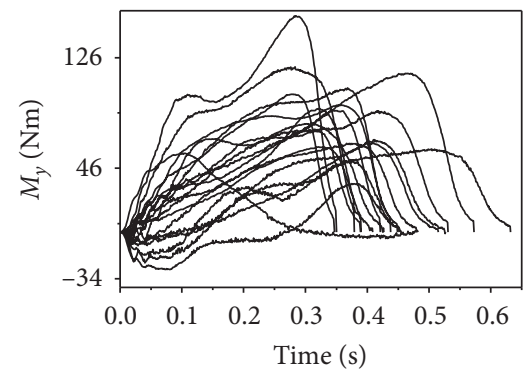

(e)

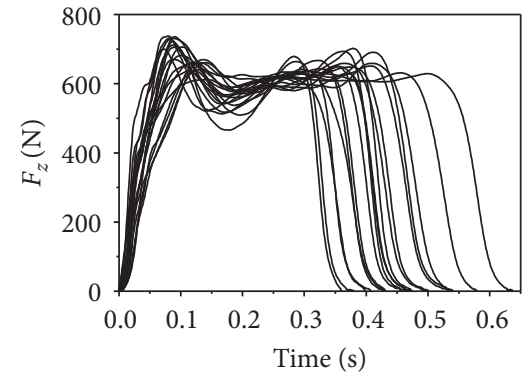

(c)

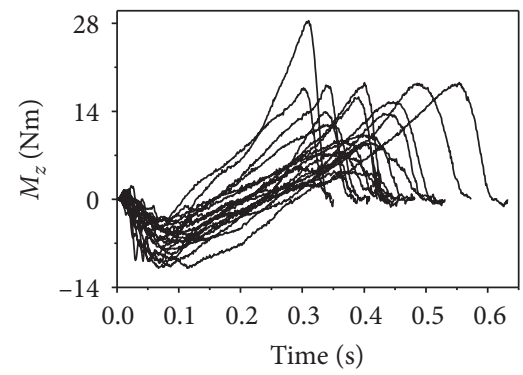

(f)

FIGURE 9: Filtering GRFs and measured GRMs.

TABLE 1: Linear fitting parameters.

\begin{tabular}{lcccccc}
\hline Peak forces & $F_{x} / G$ & $F_{y} / G$ & $F_{z} / G$ & $M_{x} / G$ & $M_{y} / G$ & $M_{z} / G$ \\
\hline Intercept & 0.16 & 0.066 & 1.04 & 0.11 & 0.14 & 0.03 \\
Slope & 0.015 & 0.006 & 0.027 & -0.025 & -0.015 & -0.004 \\
\hline
\end{tabular}

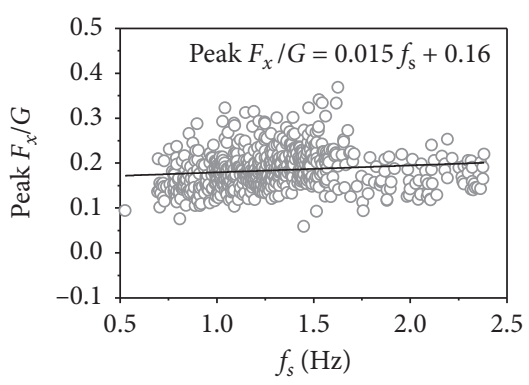

(a)

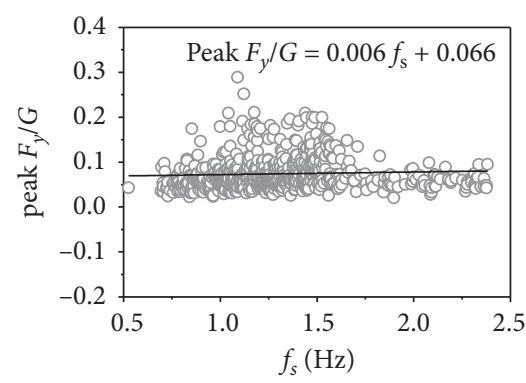

(b)

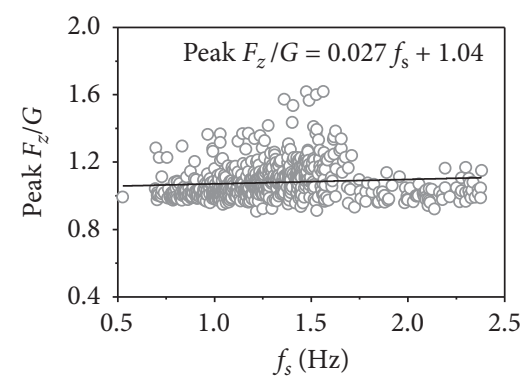

(c)

FIgURE 10: Linear fitting between step rate and normalized ground reaction forces.

always keep a negative correlation with the step rates, which means that the faster step rate results in smaller moment. The module value from the longitudinal GRMs reaches maximum, and the faster step variations would bring the weaker moment. The statistical fitting results from normalized peaks of normalized GRFs and GRMs are indicated in Figures 10 and 11, respectively.

To further extract the normalized models for these forces induced by walking pedestrians, the Fourier series in equation (17) are introduced to describe the relationship between the normalized forces divided by body weight $G$ and step rates, and its first six factors are calculated to ensure enough fitting accuracy. The $\left(\mathrm{DLF}_{\mu}^{j}\right)$ and $\left(\mathrm{DTF}_{\mu}^{j}\right)$ in equation (17) are defined as the $j^{\text {th }}$ dynamic load factor and dynamic torsional factor in the $\mu$ direction, respectively. All the factors from GRFs are statistically analyzed and linearly fitted by the step rate $\left(f_{\mathrm{s}}\right)$, whose values are listed in Table 2 . It is noted that the modular value from the 1st slope intercept in the fitting results is always larger than the other higherorder modular values The $1^{\text {st }}$ DLF from GRFs always has significant impacts on these excitation forces. In addition, the $1^{\text {st }}$ modules of slope and intercept from $\mathrm{DLF}_{z}$ also keep 


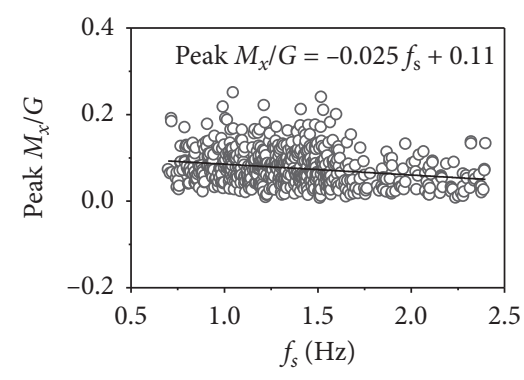

(a)

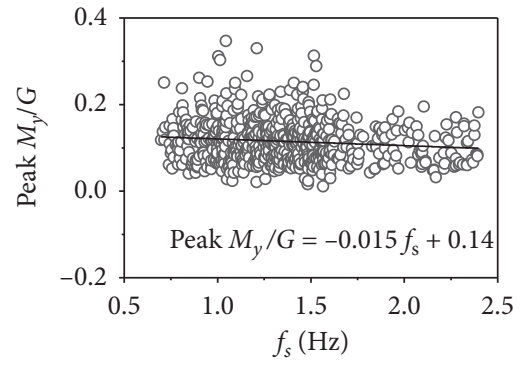

(b)

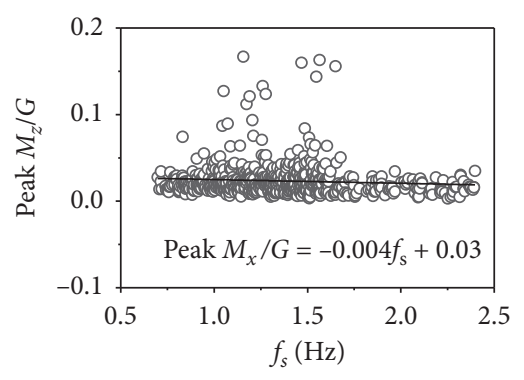

(c)

FIgURE 11: Linear fitting between step rate and normalized moments.

TABLE 2: Fitting coefficients on DLF.

\begin{tabular}{lccccc}
\hline & \multicolumn{2}{c}{ Slopes } & & \multicolumn{2}{c}{ Intercepts } \\
& $\mathrm{DLF}_{x}$ & $\mathrm{DLF}_{y}$ & $\mathrm{DLF}_{z}$ & $\mathrm{DLF}_{x}$ & $\mathrm{DLF}_{y}$ \\
\hline $1^{\text {st }}$ & -0.015 & 0.01 & -0.026 & 0.0032 & 0.028 \\
$2^{\text {nd }}$ & -0.005 & -0.004 & 0.009 & -0.1 & 0.004 \\
$3^{\text {rd }}$ & -0.002 & 0.002 & 0.05 & 0.005 & 0.006 \\
$4^{\text {th }}$ & -0.0003 & -0.004 & -0.03 & -0.033 & 0.005 \\
$5^{\text {th }}$ & 0.013 & -0.001 & -0.028 & -0.011 & 0.13 \\
$6^{\text {th }}$ & 0.006 & -0.002 & -0.038 & -0.0021 & 0.068 \\
\hline
\end{tabular}

far larger than values from $\mathrm{DLF}_{x}$ or $\mathrm{DLF}_{y}$, which implies walking pedestrian induces the most unfavorable excitation in the vertical direction:

$$
\begin{cases}\frac{F_{\mu}}{G}=\sum_{j=1}^{6} \mathrm{DLF}_{\mu}^{j} \sin \left(\frac{j \pi t}{T}\right), & (\mu=x, y), \\ \frac{M_{\mu}}{G}=\sum_{j=1}^{6} \mathrm{DMF}_{\mu}^{j} \sin \left(\frac{j \pi t}{T}\right), & (\mu=x, y, z) .\end{cases}
$$

Figure 12 gives the statistical linear fitting results based on a least-square method between step rate and first six $\mathrm{DLF}_{\mathrm{s}}$ in the $x$ direction. Its corresponding slopes are -0.015 , $-0.005,-0.002,-0.0003,0.013$, and 0.006 , respectively. The first four slopes are negative, which means that the faster walking rate would alleviate the longitudinal excitation. The corresponding first six intercepts are $0.0032,-0.1,0.005$, $-0.033,-0.011$, and -0.0021 , which are very smaller, which implies that the change of step rate has very slight impacts on the $\mathrm{GRF}_{x}$. Similarly, Figure 13 presents the linear fitting formulas between the first six $\mathrm{DLF}_{\mathrm{s}}$ and step rate, and the corresponding slopes are $0.01,-0.004,0.002,-0.004,-0.001$, and -0.002 , respectively. The first positive slope is far larger than the other five slopes, which means that the faster walking rate would promote the lateral excitation. The corresponding first six intercepts are 0.028, 0.004, 0.006, $0.005,-0.005$, and 0.002 . Similarly, the first value is far larger than others. This once again implies that the main lateral excitation is contributed by the first DLF. The statistical linear fitting on the vertical DLFs in Figure 14 indicates that the first value of intercepts with 1.139 is far larger than the last five corresponding values and main vertical excitation induced by the first DLF. The first slope on the vertical DLF is -0.026 , which implies the increase of step rate tends to a slight decrease in the vertical excitation.

All the factors from GRMs are statistically analyzed and linearly fitted by the step rate $\left(f_{\mathrm{s}}\right)$, whose values are listed in Table 3 . It is noted that the module value from the $1^{\text {st }}$ slope whether intercept always keeps maximum than the other higher-order modules. The $1^{\text {st }}$ DMF from GRMs always has significant impacts on these excitation forces. In addition, the $1^{\text {st }}$ modules of slope and intercept from $\mathrm{DMF}_{x}$ and $\mathrm{DMF}_{y}$ keep far larger than values from $\mathrm{DMF}_{z}$, which implies walking pedestrian induces the most unfavorable torsional excitation in longitudinal and lateral directions. The statistical fitting on the $\mathrm{DMF}_{x}$ is shown in Figure 15, and the first six slopes are $-0.029,0.003,-0.003,-0.002,-0.001$, and -0.003 , respectively. The corresponding intercepts are 0.11 , $-0.006,0.02,0.003,0.0008$, and 0.005 . The first values of both the slopes and intercepts are far larger than other values, which means the $\mathrm{DMF}_{x}^{1}$ plays a control role with the $\mathrm{GRM}_{x}$. The faster step rate tends to alleviate the torsional excitation in the longitudinal direction. Figure 16 continuously plots the estimated fitting results on the first six $\mathrm{DMF}_{y}$ of the lateral moment $\mathrm{GRM}_{y}$ and the corresponding slopes are $-0.016,0.006,0.001,0.0017,-0.001$, and -0.002 , respectively. The first six intercepts are 0.097, $-0.015,0.011,0.0005$, -0.0031 , and 0.004 . Similarly, the first values from both the slopes and intercepts are far larger than the other behind values, as well as the $\mathrm{DMF}_{y}^{1}$ plays a control role on the lateral torsional excitation. The faster step rate tends to alleviate the excitation in the lateral direction. Besides, the fitting approximation on the vertical moment in Figure 17 also presents the first six slopes corresponding to the values $-0.001,0.0008,0.0004,-0.0004,0.0005$, and 0.0002 , which 


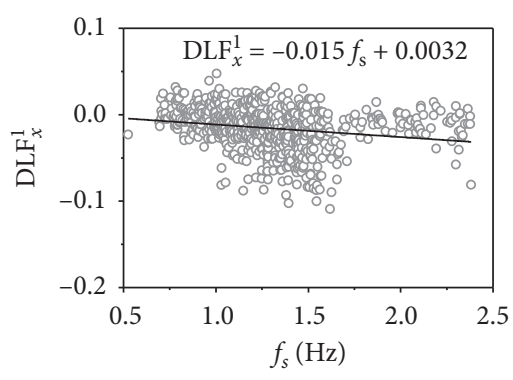

(a)

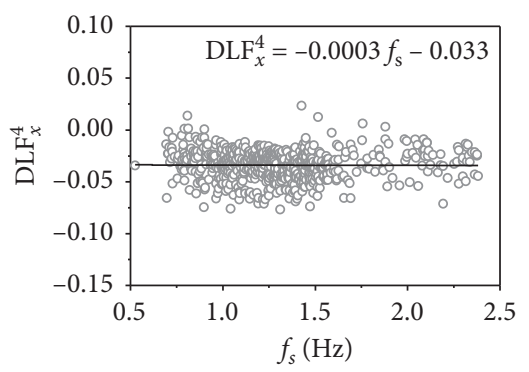

(d)

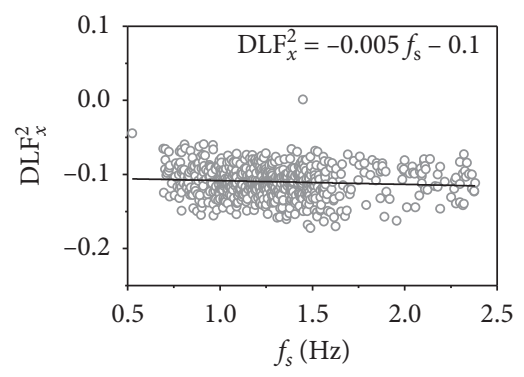

(b)

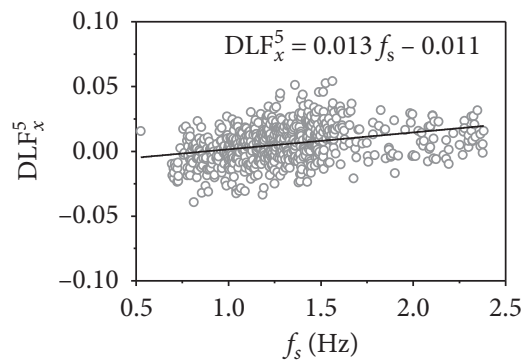

(e)

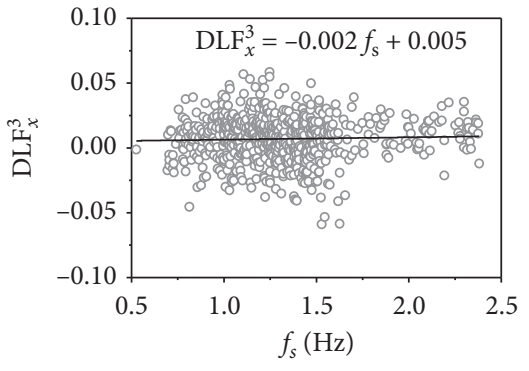

(c)

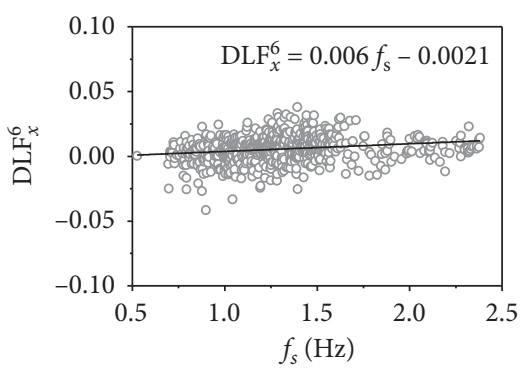

(f)

FIgURE 12: The fitting relationship between $\operatorname{DLF}_{x}^{j}$ and $f_{s}$.

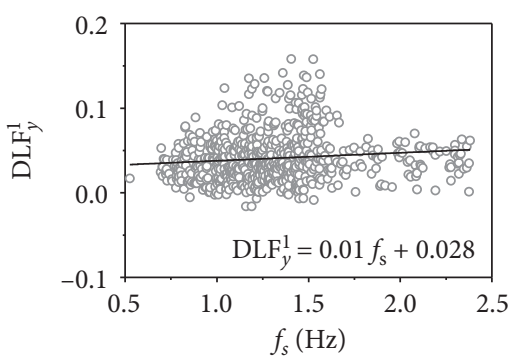

(a)

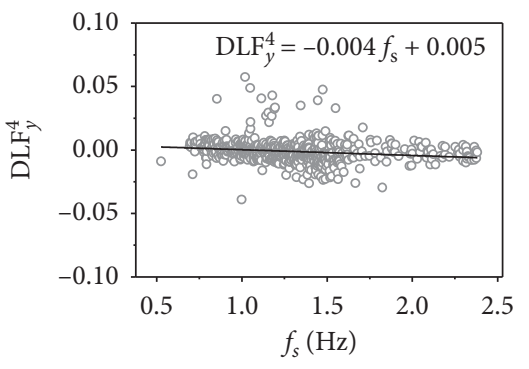

(d)

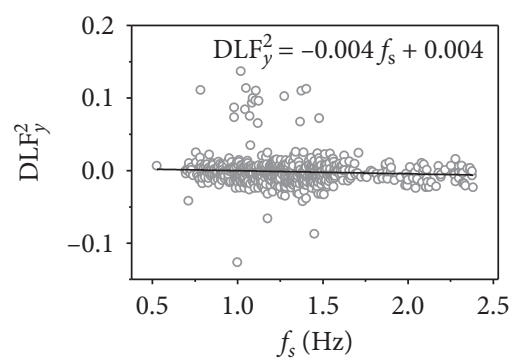

(b)

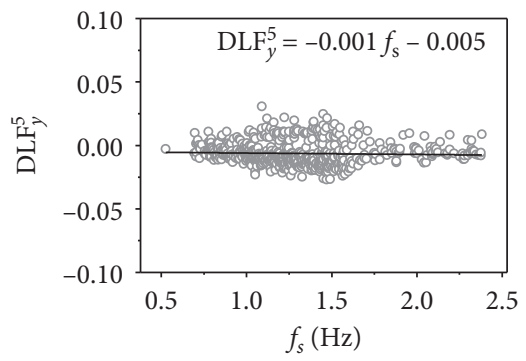

(e)

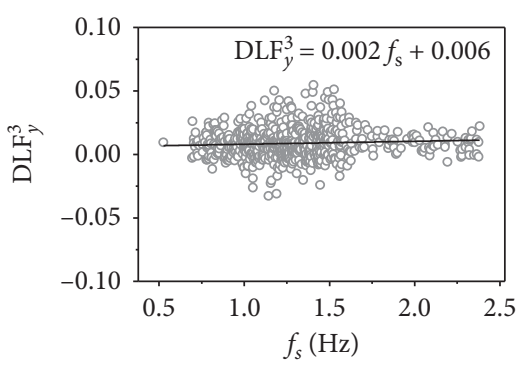

(c)

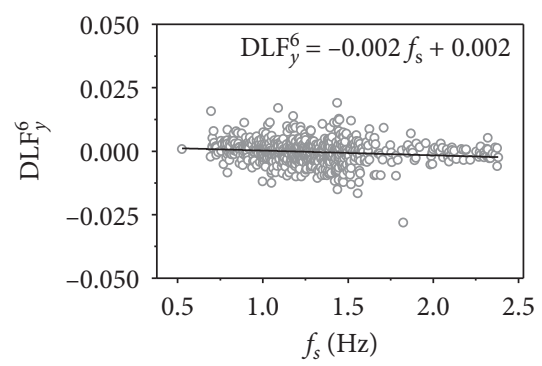

(f)

FIGURE 13: The fitting relationship between $\operatorname{DLF}_{y}^{j}$ and $f_{s}$.

are very puny so that the variation of step rate has no effects on the moment excitation. Comparing with the longitudinal DMF $x$ and lateral $\mathrm{DMF}_{y}$, the intercepts from the vertical $\mathrm{DMF}_{z}$ are also very tiny and its maximum occurs on the first value of 0.007 . The matching between the above fitting models with measurements indicates the proposed Fourier series models could well describe the excitation forces and moments from walking foot. Figure 18 gives the typical matching results between the fitting curves and measurements with a participant with $49 \mathrm{~kg}$ mass and $166 \mathrm{~cm}$ height.

\section{Remarks and Conclusions}

The proposed Kalman filter is developed based on the linear governing equation, so it only can deal with the linear filtering problems. It is noted that the human mass, leg 


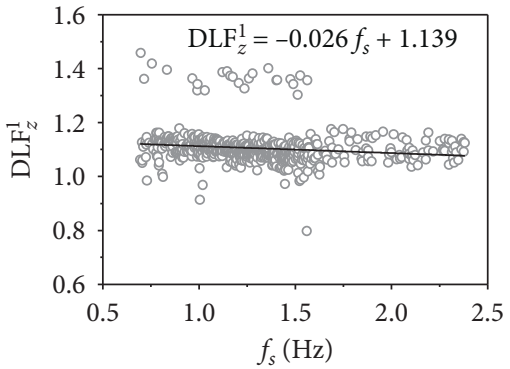

(a)

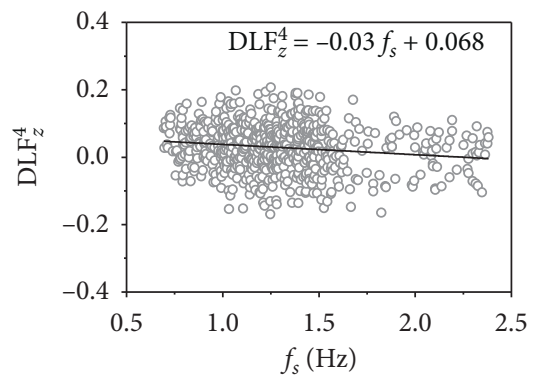

(d)

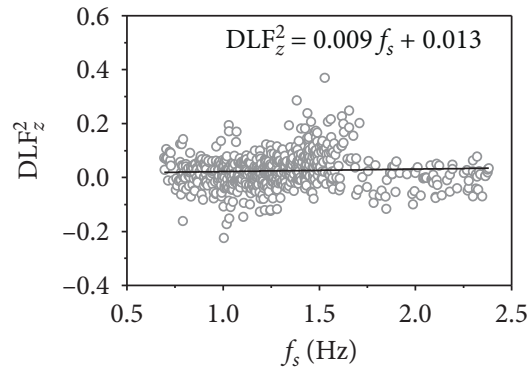

(b)

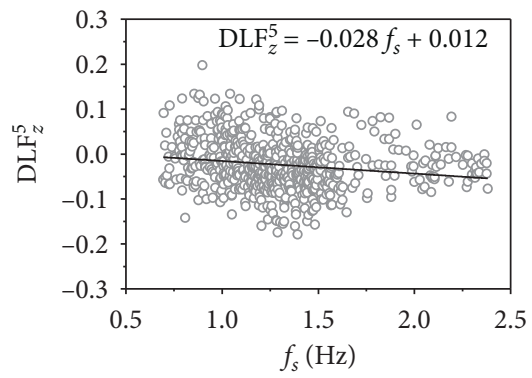

(e)

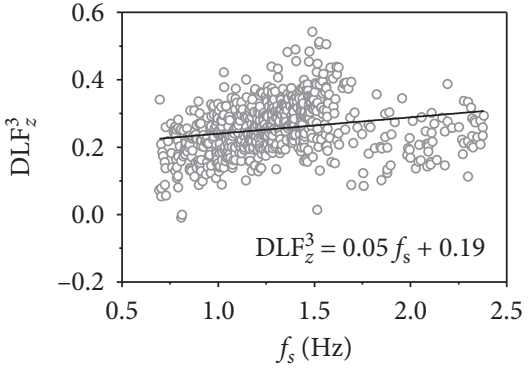

(c)

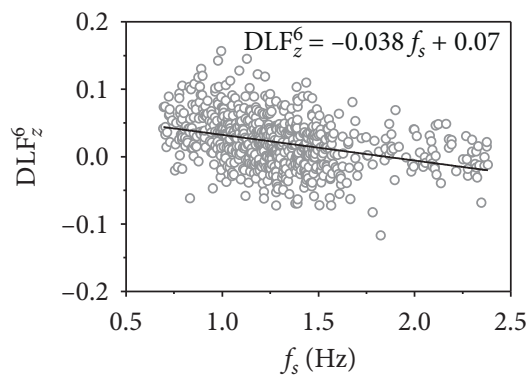

(f)

FIgURE 14: The fitting relationship between $\operatorname{DLF}_{z}^{j}$ and $f_{s}$.

TABLE 3: Fitting coefficients on DMFs.

\begin{tabular}{|c|c|c|c|c|c|c|}
\hline & \multicolumn{3}{|c|}{ Slopes } & \multicolumn{3}{|c|}{ Intercepts } \\
\hline & $\mathrm{DMF}_{x}$ & $\mathrm{DMF}_{y}$ & $\mathrm{DMF}_{z}$ & $\mathrm{DMF}_{x}$ & $\mathrm{DMF}_{y}$ & $\mathrm{DMF}_{z}$ \\
\hline $1^{\text {st }}$ & -0.029 & -0.016 & -0.001 & 0.11 & 0.097 & 0.007 \\
\hline $2^{\text {nd }}$ & 0.003 & 0.006 & -0.0008 & -0.006 & -0.015 & -0.0008 \\
\hline $3^{\mathrm{rd}}$ & -0.003 & 0.001 & 0.0004 & 0.02 & 0.011 & 0.0008 \\
\hline $4^{\text {th }}$ & -0.002 & -0.0005 & -0.0004 & 0.003 & 0.0005 & -0.00003 \\
\hline $5^{\text {th }}$ & -0.001 & 0.0017 & 0.0005 & 0.0008 & -0.0031 & -0.0005 \\
\hline $6^{\text {th }}$ & -0.003 & -0.002 & 0.0002 & 0.005 & 0.004 & 0.0002 \\
\hline
\end{tabular}

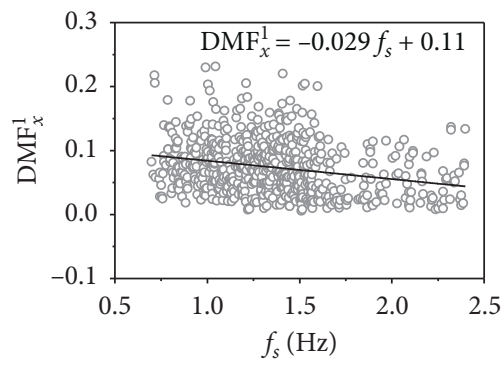

(a)

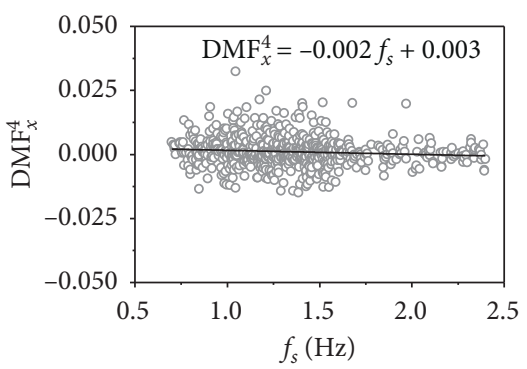

(d)

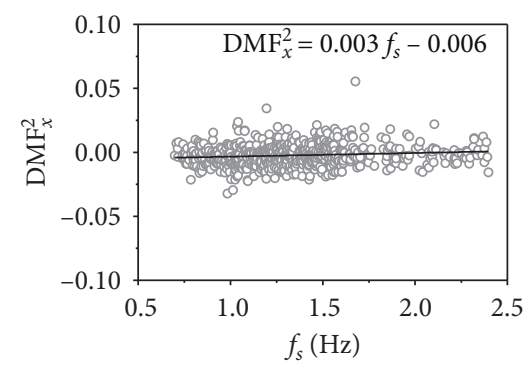

(b)

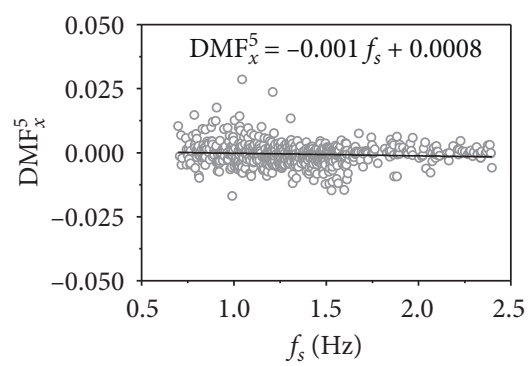

(e)

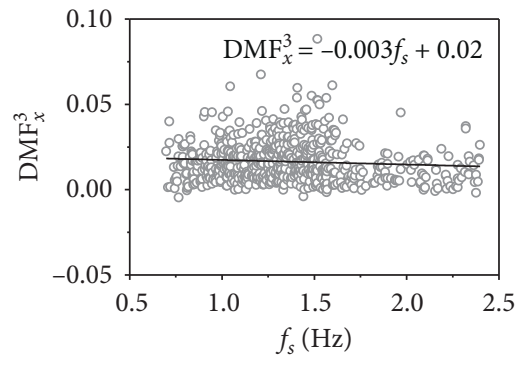

(c)

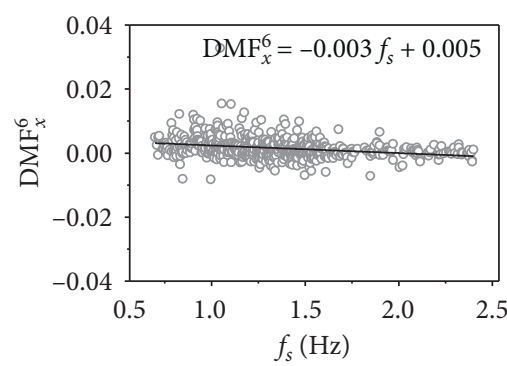

(f)

FIGURE 15: The fitting relationship between $\mathrm{DMF}_{x}^{j}$ and $f_{s}$. 


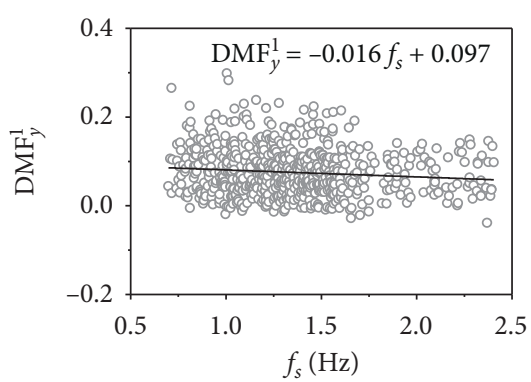

(a)

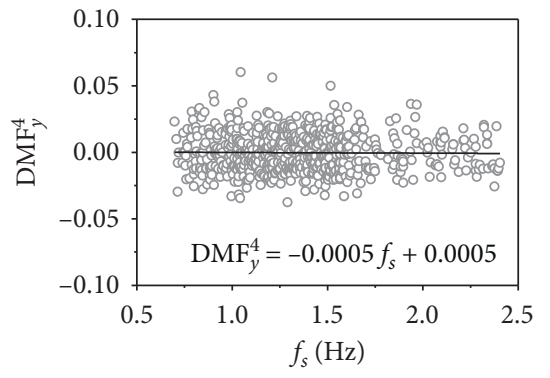

(d)

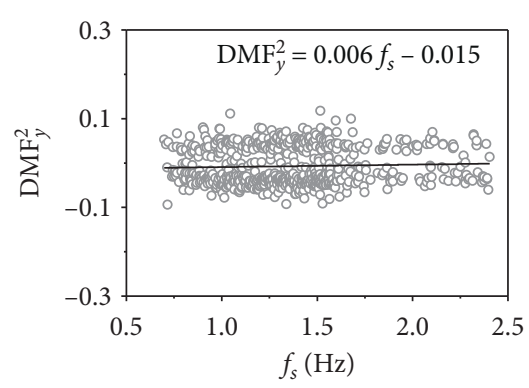

(b)

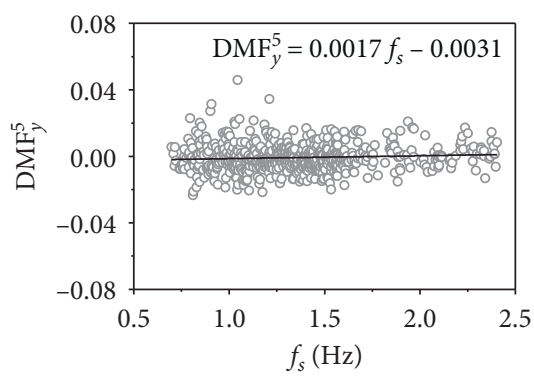

(e)

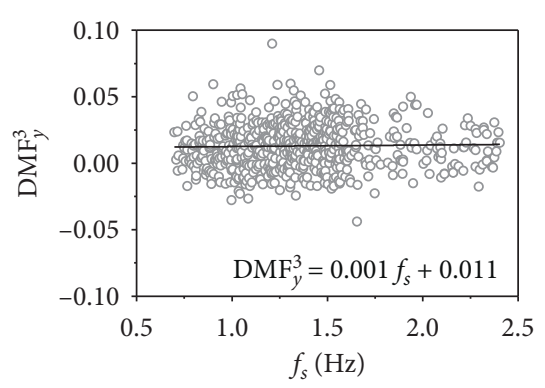

(c)

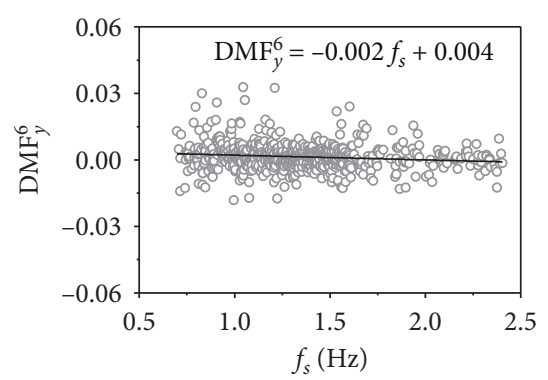

(f)

FIGURE 16: The fitting relationship between $\mathrm{DMF}_{y}^{j}$ and $f_{s}$.

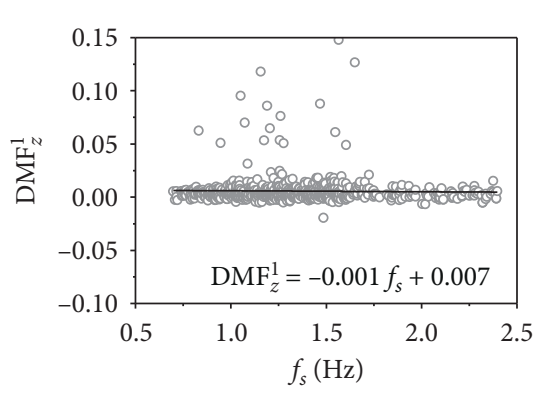

(a)

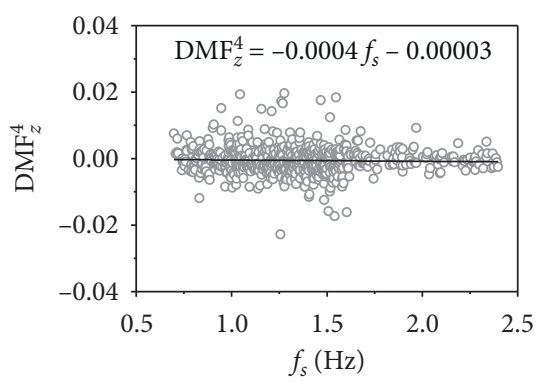

(d)

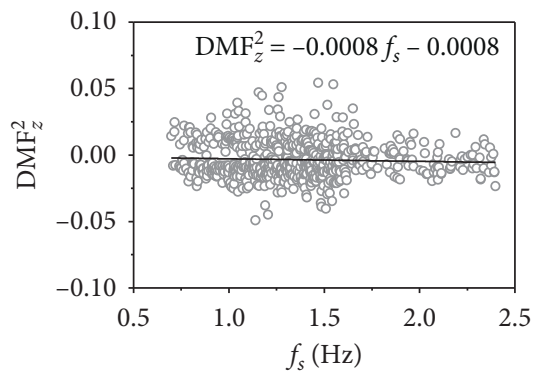

(b)

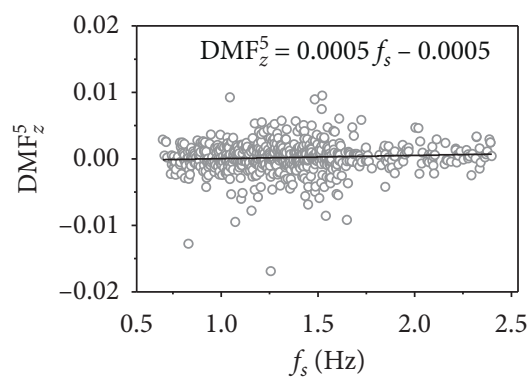

(e)

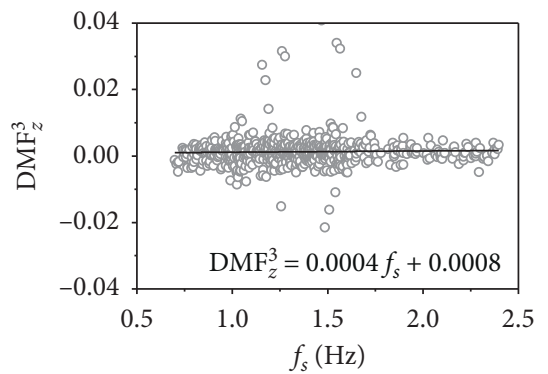

(c)

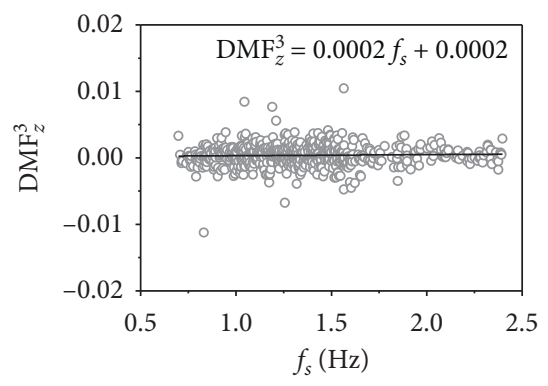

(f)

Figure 17: The fitting relationship between $\mathrm{DMF}_{z}^{j}$ and $f_{s}$.

damping ratio, leg stiffness, and time interval are need to be predefined and input. The repeated filtering process verifies that the slight variables on leg damping ratio and stiffness have no influences on the filter results with the measured GRFs and it has robust stability. However, it is also noted that the value of leg damping ratio and stiffness should be defined as a reasonable range which can refer to the measured body parameters [18]. In addition, the proposed method only can deal with GRFs rather than GRMs, as it is developed on the governing equation with GRFs. Further, it can be developed to treat with the measured GRMs based on a suitable governing equation with GRMs. Moreover, the proposed Kalman filter only can handle the data in the time domain rather than the frequency domain, which would be further developed to treat with a frequency domain in future.

The movements of walking crowd have considerable complexity, and their walking behaviors also have induced tough serviceable challenges on these slender structures. This 


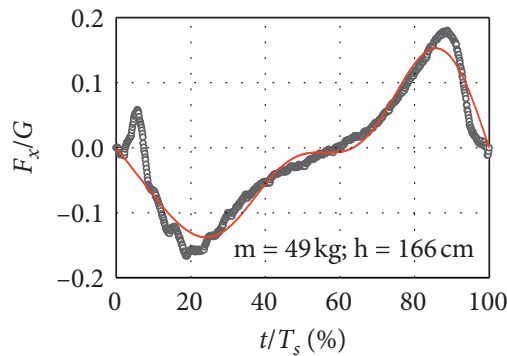

○ Measurement _ Fitting

(a)

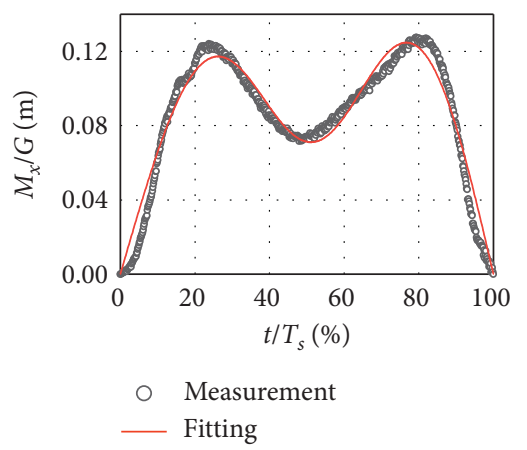

(d)

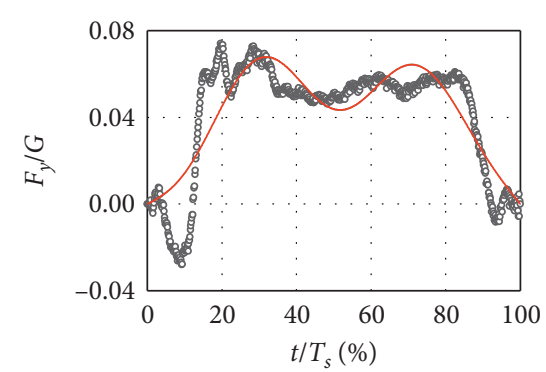

- Measurement Fitting

(b)

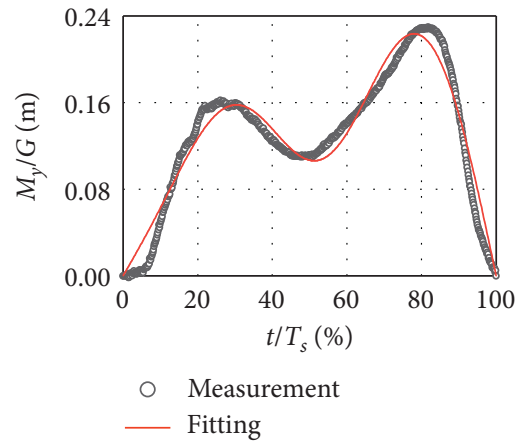

(e)

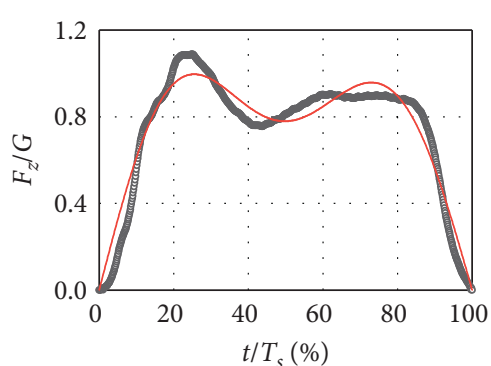

o Measurement __ Fitting

(c)

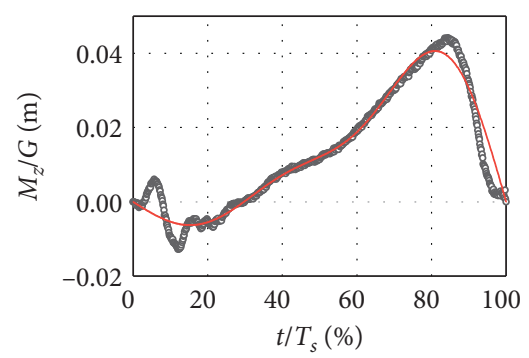

○ Measurement

_ Fitting

(f)

FIgURE 18: The fitting results of the participant with $49 \mathrm{~kg}$ mass and $166 \mathrm{~cm}$ height.

work tries to explore the excitation mechanism from the ground reaction forces and movements by the measurements on a case sample comprised of 36 walking pedestrians. Then, a noise-immune Kalman filter is proposed to the treatment on the original ground reaction forces. The applications of the bipedal walking model and measured GRFs confirm that the Kalman filter can well reduce the measured noises. Further study is implemented. The Fourier series are employed to describe the excitation models on the measured GRFs and GRMs, whose first six factor coefficients are statistically fitted. A novel result shows that pedestrian foot mainly generates longitudinal and lateral torsional movements, and the vertical torsional movement is comparatively very slight. This work comprehensively explores the potential full-space loads induced by walking pedestrian, and it can provide references for the design of these slender structures such as footbridges, grandstands, or stations under crowd excitation.

\section{Appendix}

In order to present clearly the data processing framework, the corresponding key equations on the Kalman filter process are shown as follows.

Firstly, the system prior state $\widehat{X}_{i+1 \mid i}$ of the vector $X$ with the next moment point based on the current moment state $\widehat{X}_{i \mid i}$ can be estimated as follows:

$$
\widehat{X}_{i+1 \mid i}=\Psi \widehat{X}_{i \mid i}+\Gamma f_{i} .
$$

Then, the prior estimation error vector $\varepsilon_{i+1 \mid i}$ is the differences between the system vector $X_{i+1}$ and the prior state $\widehat{X}_{i+1 \mid i}$, which can be obtained as follows:

$$
\varepsilon_{i+1 \mid i}=X_{i+1}-\widehat{X}_{i+1 \mid i}=\Psi \varepsilon_{i \mid i}+w_{i} .
$$

The prior estimation covariance matrix $P_{i+1 \mid i}$ is the expectation value of the product of both $\varepsilon_{i+1 \mid i}$ and $\varepsilon_{i+1 \mid i}^{T}$, which can be calculated as follows:

$$
P_{i+1 \mid i}=E\left(\varepsilon_{i+1 \mid i} \varepsilon_{i+1 \mid i}^{T}\right)=\boldsymbol{\Psi} P_{i \mid i} \Psi^{T}+\mathbf{Q}_{i}
$$

The system posterior state vector $\hat{X}_{i+1 \mid i+1}$ is the sum between the system prior state vector $\widehat{X}_{i+1 \mid i}^{i+1 \mid i+1}$ and an increment induced by the contribution induced by a gain term related to a gain matrix $\mathbf{K}_{i+1}$ in equation (A.8), which can be estimated as follows:

$$
\widehat{X}_{i+1 \mid i+1}=\widehat{X}_{i+1 \mid i}+\mathbf{K}_{i+1}\left[F_{i+1}-\mathbf{H} \widehat{X}_{i+1 \mid i}\right] .
$$

The posterior estimation error vector $\varepsilon_{i+1 \mid i+1}$ means the difference between $X_{i+1}$ and the posterior state vector $\widehat{X}_{i+1 \mid i+1}$ in equation (A.4) which can be obtained as

$$
\varepsilon_{i+1 \mid i+1}=X_{i+1}-\widehat{X}_{i+1 \mid i+1}=\left(I-\mathbf{K}_{i+1} \mathbf{H}\right) \varepsilon_{i+1 \mid i}-\mathbf{K}_{i+1} v_{i+1} .
$$

Similar to equation (A.3), the posterior estimation covariance $P_{i+1 \mid i+1}$ is the expectation value of the product of both the posterior estimation error vector $\varepsilon_{i+1 \mid i+1}$ and its corresponding transpose $\varepsilon_{i+1 \mid i+1}^{T}$, which can be calculated as follows: 


$$
\begin{aligned}
P_{i+1 \mid i+1} & =E\left(\varepsilon_{i+1 \mid i+1} \varepsilon_{i+1 \mid i+1}^{T}\right), \\
& =\left(I-\mathbf{K}_{i+1} \mathbf{H}\right) P_{i+1 \mid i}\left(I-\mathbf{H}^{T} \mathbf{K}_{i+1}^{T}\right)+\mathbf{K}_{i+1} \mathbf{R}_{i+1} \mathbf{K}_{i+1}^{T} .
\end{aligned}
$$

The gain matrix $\mathbf{K}_{i+1}$ appeared in equations (A.4 A.6) can be obtained by finding the extreme point for the derivation of the trace of the posterior estimation covariance $P_{i+1 \mid i+1}$ as follows:

$$
\frac{\partial \operatorname{tr}\left(P_{i+1 \mid i+1}\right)}{\partial \mathbf{K}_{i+1}}=2 \mathbf{K}_{i+1}\left(\mathbf{H} P_{i+1 \mid i} \mathbf{H}^{T}+\mathbf{R}_{i+1}\right)-2 P_{i+1 \mid i} \mathbf{H}^{T}=0 .
$$

The gain matrix $\mathbf{K}_{i+1}$ can be obtained as equation (A.8) by solving equation (A.7):

$$
\mathbf{K}_{i+1}=P_{i+1 \mid i} \mathbf{H}^{T}\left(\mathbf{H} P_{i+1 \mid i} \mathbf{H}^{T}+\mathbf{R}_{i+1}\right)^{-1} .
$$

The filtering result can be calculated by the following equation:

$$
F=\mathbf{H} \widehat{X}_{i+1 \mid i+1} .
$$

\section{Data Availability}

All relevant data underlying this research are included in this paper. As the data were from the cooperative experiments conducted by multiple stakeholders, with restrictions on public usage, the authors cannot provide more public access to the data and experiments.

\section{Conflicts of Interest}

The authors declare that they have no conflicts of interest.

\section{Acknowledgments}

The study was supported by the National Natural Science Foundation of China (nos. 51808247, 51808246, 11902123, and 51904113), Huaiyin Institute of Technology Laboratory Open Fund (nos. JSZP201901 and JSZP201902), Jiangsu Natural Science Foundation (nos. 18KJB560003 and BK20181061), Science and Technology Projects of Monastery of Housing and Urban-Rural Development of the People's Republic of China (no. 2017K6004), Huaian Natural Science Research Project (no. HAB201836), and Open Fund of State Key Laboratory of Coastal and Offshore Engineering (no. LP1829).

\section{References}

[1] J. H. Rainer and G. Pernica, "Vertical dynamic forces from footbridges," Canadian Acoustics, vol. 14, no. 2, pp. 12-21, 1986.

[2] A. Ebrahimpour, A. Hamam, R. L. Sack, and W. N. Patten, "Measuring and modeling dynamic loads imposed by moving crowds," Journal of Structural Engineering, vol. 122, no. 12, pp. 1468-1474, 1996.

[3] Q. Li, J. Fan, J. Nie, Q. Li, and Y. Chen, "Crowd-induced random vibration of footbridge and vibration control using multiple tuned mass dampers," Journal of Sound and Vibration, vol. 329, no. 19, pp. 4068-4092, 2010.
[4] J. S. Fan, Y. Chen, and J. G. Nie, "Modeling and confirmation of pedestrian induced walking load on footbridge," Chinese Journal of Computational Mechanics, vol. 29, no. 4, pp. 538-544, 2012.

[5] S. C. Kerr and N. W. M. Bishop, "Human induced loading on flexible staircases," Engineering Structures, vol. 23, no. 1, pp. 37-45, 2001.

[6] S. M. Hsiang and C. Chang, "The effect of gait speed and load carrying on the reliability of ground reaction forces," Safety Science, vol. 40, no. 7-8, pp. 639-657, 2002.

[7] J. Kala, V. Salajka, and P. Hradil, "Footbridge response on single pedestrian induced vibration analysis," World Academy of Science, vol. 50, pp. 744-755, 2009.

[8] F. Ricciardelli and A. D. Pizzimenti, "Lateral walking-induced forces on footbridges," Journal of Bridge Engineering, vol. 12, no. 6, pp. 677-688, 2007.

[9] E. T. Ingólfsson, C. T. Georgakis, F. Jönsson, and J. Jonsson, "Experimental identification of pedestrian-induced lateral forces on footbridges," Journal of Sound and Vibration, vol. 330, no. 6, pp. 1265-1284, 2011.

[10] M. Bocian, J. H. G. Macdonald, J. F. Burn, and D. Redmill, "Experimental identification of the behaviour of and lateral forces from freely-walking pedestrians on laterally oscillating structures in a virtual reality environment," Engineering Structures, vol. 105, pp. 62-76, 2015.

[11] S. Erlicher, A. Trovato, and P. Argoul, "Modeling the lateral pedestrian force on a rigid floor by a self-sustained oscillator," Mechanical Systems and Signal Processing, vol. 24, no. 5, pp. 1579-1604, 2010.

[12] S. E. Mouring and B. R. Ellingwood, "Guidelines to minimize floor vibrations from building occupants," Journal of Structural Engineering, vol. 120, no. 2, pp. 507-526, 1994.

[13] L. Pedersen and C. Frier, "Sensitivity of footbridge vibrations to stochastic walking parameters," Journal of Sound and Vibration, vol. 329, no. 13, pp. 2683-2701, 2010.

[14] E. Shahabpoor, A. Pavic, and V. Racic, "Identification of massspring-damper model of walking humans," Structures, vol. 5, pp. 233-246, 2016.

[15] L. Cai, Z. Zhang, J. Yang, Y. Yu, T. Zhou, and J. Qin, “A noiseimmune Kalman filter for short-term traffic flow forecasting," Physica A: Statistical Mechanics and Its Applications, vol. 536, p. 9, Article ID 122601, 2019.

[16] Y.-A. Gao, Q.-S. Yang, and Y. Dong, "A three-dimensional pedestrian-structure interaction model for general applications," International Journal of Structural Stability and Dynamics, vol. 18, no. 9, p. 27, Article ID 1850107, 2018.

[17] Y.-A. Gao, Q.-S. Yang, Y. Dong, C. Chen, and T.-P. Ye, "Dynamic behavior of slab induced by pedestrian traffic," International Journal of Structural Stability and Dynamics, vol. 19, no. 12, Article ID 1950154, 2019.

[18] D. Z. Han, D. Zhou, and T. Ji, "Mechanical parameters of standing body and applications in human-structure interaction," International Journal of Applied Mechanics, vol. 9, no. 2, pp. 1750021-30, 2017. 